\title{
Analysis of Multi Aerial Targets Threaten Degree on Terminal Defense System
}

\author{
L.G. Chen \\ Systems Engineering Research Institute \\ China
}

\author{
Y. Zhang \\ Northwestern Polytechnical University \\ China
}

\author{
X.L. Liu \\ Northwestern Polytechnical University \\ China
}

\begin{abstract}
The characteristics of incoming aerial target are multi batches and multi azimuths in modern war. The influence of target threaten degree have many kinds of factors also. Due to this, the paper analysed the factors influencing the threatendegree of aerial targetsand established the membership functions of each factor. The article put forward a kind of improved grey comprehensive relational analysis method. Finally, the paper verified the accuracy and effectiveness of the method through an example.
\end{abstract}

Keywords-hreaten degree,grey comprehensive relational analysis, terminal defense system.

\section{INTRODUCTION}

With the application and the development of high technology in the military, the major operational means of modern war is air raid. The terminal defensesystem[1] is in order to prevent our position from air raid. However, the characteristics of incoming aerial target are multi batches and multi azimuths, the command and control center make the correct and reasonable fire distribution[2] is very important.The decision of fire distribution is based on the threaten degree. The threaten degree is make sure the quantitative of target threaten, and the biggest is the major operational goal.The article put forward a kind of improved grey comprehensive[3] relational analysis method. The paper finished four jobs. First, analysed the threaten factors of the incoming target; Secondly, constructed a specialmembershipfunction[4] to confirm the threaten degree of each factors; Then, we gave the operation process of the grey comprehensive relational analysis; Finally, we verified the accuracy and the effectiveness of the method through an example.

\section{THREATEN DEGREE EVALUATION AND ANYLYSIS}

\section{A. The Influence factors of the Threaten Degree}

A lot of scholarsconsidered course short is ainfluence factor of the threaten degree in the past years. However, we can't know the course short in terminal defense system, but we can estimate the course short according to the current distance and the relative course angle, as shown in Fig1.In this diagram, $\lambda$ is the relative course angle, $d$ is the distance and $\mathrm{p}$ is the course short. So, we can estimate p from Figure 1:

$$
p=d \sin (\lambda)
$$

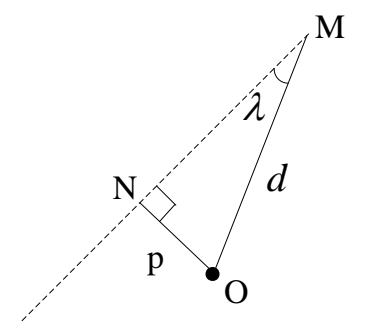

FIGURE I. THE RELATIONS BETWEEN AND AMONG DISTANCE, RELATIVE COURSE ANGLE

and course short

According to Fig 1,we can know that the course short is not a independent factor, it will cause error to some extent. Therefore, the factors which influence the threaten degree in terminal defense system include distance, flying speed, flying height and the relative course angle. And the factors must follow the following principles.

1) Distance: The closer distance has bigger threaten degree.

2) Flying speed: The faster flying speed has bigger threaten degree.

3) Flying height: The lower flying height has bigger threaten degree.

4) Relative course angle: It refers to the angle between the direction of fly

and the line which is drew from target and our system. The relative course angle is smaller, the attacking intention is more obvious, so the threaten degree is bigger.

\section{B. The Construction ofMembership Function}

1) Distance: In general, The closer distance has bigger threaten degree. And we consider that the threaten degree is 0 
when the distance is greater than $10000 \mathrm{~m}$ in terminal defense system. So the membership function of distance can be eqn (2):

$$
\mu(d)= \begin{cases}e^{-\alpha d^{2}} & , d<10000 \\ 0 & , d \geq 10000\end{cases}
$$

In eqn (2), $d$ is the distance, $\alpha$ is constant, it is equal to $0.001 m^{-2}$.

2) Flying speed:The membership function of flying spped can be eqn (3):

$$
\mu(v)=1-e^{-\alpha v}
$$

In eqn (3), $v$ is the flying speed, $\alpha$ is constant, it is equal to $0.005 \mathrm{~s} / \mathrm{m}$.

3) Flying height: In general, Thelower flying height has bigger threaten degree. And we consider that the threaten degree is 0 when the flying height is greater than $1000 \mathrm{~m}$ in terminal defense system. So the membership function of distance can be eqn (4):

$$
\mu(h)= \begin{cases}e^{-\alpha h^{2}}, & h>1 k m \\ 1, & h \leq 1 k m\end{cases}
$$

In eqn (4), $h$ is the flying speed, $\alpha$ is constant, it is equal to $0.005 \mathrm{~m}^{-2}$

4) Relative course angle: when the relative course angle is less than $\frac{\pi}{2}$, the target does have some threaten degree, and it will become bigger with the relative course angle become littler. In addition, we consider that the threaten degree is 0 when the relative course angle is more than $\frac{\pi}{2}$. So the membership function of relative course angle can be eqn(5):

$$
\mu(\lambda)= \begin{cases}e^{\alpha \lambda}, & \lambda \leq \frac{\pi}{2} \\ 0, & \lambda>\frac{\pi}{2}\end{cases}
$$

In eqn (5), $\lambda$ is the relative course angle, $\alpha$ is constant, it is equal to -0.005 .

\section{Calculation of the Threaten Degree}

Define a single target threaten degree vector is $X_{i}$. We can calculate the threaten degree of each influent factors using the membership function in the section 2.2.

$$
X_{i}=\left[\left(\mu_{i}(d), \mu_{i}(v), \mu_{i}(h), \mu_{i}(\lambda)\right]\right.
$$

Hypothesize the total number of the target is $n$, so the decision matrix A can be expressed by eqn(7).

$$
A=\left(X_{0}, X_{1}, \ldots, X_{n-1}\right)^{T}=\left[\begin{array}{cccc}
x_{0}(1) & x_{0}(2) & x_{0}(3) & x_{0}(4) \\
x_{1}(1) & x_{1}(2) & x_{1}(3) & x_{1}(4) \\
\ldots & \ldots & \ldots & \ldots \\
x_{n-1}(1) & x_{n-1}(2) & x_{n-1}(3) & x_{n-1}(4)
\end{array}\right]_{n \times 4}(7)
$$

Find the maximum value of each row in matrix A to constitute the ideal optimal reference sequence $\alpha$, and find the minimum value of each row in matrix A to constitute the ideal worst reference sequence $\beta$.

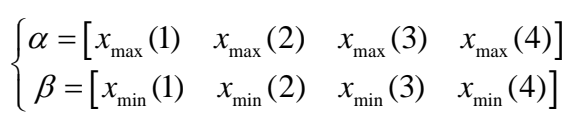

Extend the $\alpha$ and $\beta$ to matrix which is the same in each row. So the difference matrix $\mathrm{B}$ of the $\alpha$ can be confirm by eqn(9) and the difference matrix $\mathrm{C}$ of the $\beta$ can be confirm by eqn(10).

$$
\begin{aligned}
B & =\left[\begin{array}{cccc}
x_{\max }(1) & x_{\max }(2) & x_{\max }(3) & x_{\max }(4) \\
x_{\max }(1) & x_{\max }(2) & x_{\max }(3) & x_{\max }(4) \\
\cdots & \ldots & \ldots & \ldots \\
x_{\max }(1) & x_{\max }(2) & x_{\max }(3) & x_{\max }(4)
\end{array}\right]-A \\
C & =A-\left[\begin{array}{cccc}
x_{\min }(1) & x_{\min }(2) & x_{\min }(3) & x_{\min }(4) \\
x_{\min }(1) & x_{\min }(2) & x_{\min }(3) & x_{\min }(4) \\
\ldots & \ldots & \ldots & \ldots \\
x_{\min }(1) & x_{\min }(2) & x_{\min }(3) & x_{\min }(4)
\end{array}\right]_{(10)}
\end{aligned}
$$

Find the maximum value element $y_{1}$ in thematrix $\mathrm{B}$ and find the minimum value element $X_{1}$ in the matrix $\mathrm{B}$. Hypothesize any element in the matrix $\mathrm{B}$ is $b_{i j}$, so we can calculate the any element $d_{i j}$ in the correlation coefficient matrix $\mathrm{D}$ of the ideal optimal reference sequence by using eqn(11).

$$
d_{i j}=\frac{\left(x_{1}+\rho y_{1}\right)}{b_{i j}+\rho y_{1}}
$$

In eqn(11), $\rho$ is resolution ratio, it is equal to 0.5 . By the same token, we can calculate the correlation coefficient matrix $E$ of the ideal worst reference sequence by using eqn(11).

Due to the nature of the threaten factors are different, theycause different threat. So the weight in the correlation matrix of each factors are different, but the sum of weight is 1 . Given the correlation of correlation coefficient matrix of any 
target is $W_{i}=\left[r_{1}, r_{2}, r_{3}, r_{4}\right]$, we can calculate the weight of each factors by using eqn(12).

$$
w_{i}=\frac{r_{i}}{r_{1}+r_{2}+r_{3}+r_{4}}
$$

So, the final optimal relevance $\eta$ and the final worst relevance $\gamma$ of threaten degree of each target can be confirm by eqn(13).

$$
\left\{\begin{array}{l}
\eta=\sum_{k=1}^{4} w_{k} d_{0 i}(k) \\
\gamma=\sum_{k=1}^{4} w_{k} e_{0 i}(k)
\end{array}\right.
$$

The final comprehensive correlative $\delta$ of threaten degree of each target can be calculate by using eqn(14).

$$
\delta=\frac{1}{\left(1+\frac{\gamma}{\eta}\right)^{2}}
$$

The bigger $\delta$ have the greater threaten degree.

\section{THE ANALYSIS OF THE EXAMPLE}

Given the target set is $X=\left(X_{1}, X_{2}, \ldots, X_{10}\right)^{T}$, the movement information of each target showed in the Table 1.

TABLE I. MOVEMENT INFORMATION OF EACH TARGET

\begin{tabular}{|c|c|c|c|c|}
\hline Target & Distance & Flying speed & Flying height & Relative course angle \\
\hline 1 & 10 & 100 & 0.5 & 10 \\
\hline 2 & 20 & 150 & 2 & 15 \\
\hline 3 & 30 & 200 & 8 & 90 \\
\hline 4 & 25 & 500 & 10 & 50 \\
\hline 5 & 40 & 400 & 7 & 40 \\
\hline 6 & 35 & 300 & 3.5 & 30 \\
\hline 7 & 15 & 450 & 1.4 & 50 \\
\hline 8 & 50 & 250 & 2.8 & 20 \\
\hline 9 & 28 & 150 & 7.6 & 15 \\
\hline 10 & 36 & 450 & 6.8 & 52 \\
\hline
\end{tabular}

Calculate the membership value of each factor according to the membership function in the section 2.2, and show the result in the Table 2 .

TABLE II.

MEMBERSHIP VALUE OF EACH FACTOR

\begin{tabular}{|c|c|c|c|c|}
\hline Target & Distance & Flying speed & Flying height & Relative course angle \\
\hline 1 & 0.904837 & 0.393469 & 1 & 0.951229 \\
\hline 2 & 0.670320 & 0.527633 & 0.980199 & 0.927743 \\
\hline 3 & 0.406570 & 0.632121 & 0.726149 & 0.637628 \\
\hline 4 & 0.535261 & 0.917915 & 0.606531 & 0.778801 \\
\hline 5 & 0.201897 & 0.864665 & 0.782705 & 0.818731 \\
\hline 6 & 0.293758 & 0.776870 & 0.940588 & 0.860708 \\
\hline 7 & 0.798516 & 0.894601 & 0.990248 & 0.778801 \\
\hline 8 & 0.082085 & 0.713495 & 0.961558 & 0.904837 \\
\hline 9 & 0.456576 & 0.527633 & 0.749162 & 0.927743 \\
\hline 10 & 0.273624 & 0.894601 & 0.793581 & 0.771052 \\
\hline
\end{tabular}

We can knowthe ideal optimal reference sequence is [0.904837, 0.917965,1.000000,0.951229] and the ideal worst reference sequence is [0.082085, 0.393469, 0.606531, 0.637628] from the Table 2.

According to the eqn(9),the difference matrix of the ideal worst reference sequence is:

$\left[\begin{array}{llll}0.000000 & 0.527779 & 0.000000 & 0.000000 \\ 0.234517 & 0.390282 & 0.019801 & 0.023486 \\ 0.498267 & 0.285794 & 0.273851 & 0.313601 \\ 0.369576 & 0.000000 & 0.393469 & 0.172428 \\ 0.702940 & 0.053250 & 0.217295 & 0.132498 \\ 0.611079 & 0.141045 & 0.059412 & 0.090521 \\ 0.106321 & 0.023314 & 0.009752 & 0.172428 \\ 0.822752 & 0.204420 & 0.038442 & 0.046392 \\ 0.448261 & 0.390282 & 0.250838 & 0.023486 \\ 0.631213 & 0.023314 & 0.206419 & 0.180177\end{array}\right]$

The minimum value element $x_{1}$ is 0.000000 and the maximum value element $y_{1}$ is 0.822752 . So the correlation coefficient matrix of the ideal optimal reference sequence is:

$\left[\begin{array}{llll}1.000000 & 0.439588 & 1.000000 & 1.000000 \\ 0.636910 & 0.513156 & 0.954077 & 0.945992 \\ 0.452239 & 0.590066 & 0.600350 & 0.567433 \\ 0.526762 & 1.000000 & 0.511125 & 0.704647 \\ 0.369174 & 0.885392 & 0.654358 & 0.756381 \\ 0.402341 & 0.744678 & 0.873803 & 0.819642 \\ 0.794627 & 0.946366 & 0.976843 & 0.704647 \\ 0.333333 & 0.668039 & 0.914539 & 0.898656 \\ 0.478546 & 0.513156 & 0.621213 & 0.945992 \\ 0.394572 & 0.946366 & 0.665878 & 0.695417\end{array}\right]$

So, the weight of each factor can be calculate by using eqn(12) and show the result in the Table 3.

TABLE III. THE WEIGHT OF EACH FACTOR

\begin{tabular}{|c|c|c|c|c|}
\hline Target & Distance & Flying speed & Flying height & Relative course angle \\
\hline 1 & 0.290732 & 0.127804 & 0.290732 & 0.290732 \\
\hline 2 & 0.208814 & 0.168240 & 0.312798 & 0.310148 \\
\hline 3 & 0.204625 & 0.266988 & 0.271641 & 0.256747 \\
\hline 4 & 0.192071 & 0.364626 & 0.186370 & 0.256933 \\
\hline 5 & 0.138510 & 0.332192 & 0.245510 & 0.283788 \\
\hline 6 & 0.141646 & 0.262168 & 0.307627 & 0.288559 \\
\hline 7 & 0.232179 & 0.276514 & 0.285419 & 0.205888 \\
\hline 8 & 0.118431 & 0.237351 & 0.324931 & 0.319287 \\
\hline 9 & 0.187012 & 0.200537 & 0.242765 & 0.369686 \\
\hline 10 & 0.146017 & 0.350216 & 0.246418 & 0.257349 \\
\hline
\end{tabular}

So the final optimal relevance is:

$$
\left[\begin{array}{lllll}
0.928377 & 0.811160 & 0.558846 & 0.742107 & 0.720558 \\
0.757541 & 0.870067 & 0.782128 & 0.692929 & 0.732096
\end{array}\right]
$$

By the same token, we can calculate the final worst relevance is: 


$\left[\begin{array}{lllll}0.702494 & 0.595978 & 0.779754 & 0.742104 & 0.679054 \\ 0.600820 & 0.557538 & 0.728892 & 0.666759 & 0.664497\end{array}\right]$

According to the eqn(14), the final comprehensive correlative is :

$$
\left[\begin{array}{lllll}
0.324048 & 0.332307 & 0.174294 & 0.250001 & 0.265047 \\
0.311015 & 0.371441 & 0.267926 & 0.259716 & 0.274787
\end{array}\right]
$$

Show the result of the analysis of threaten degree on the Figure 2.

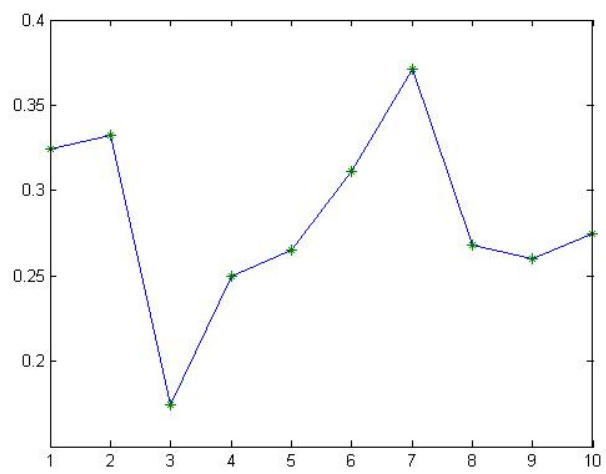

FIGURE II. THE RESULT OF THE ANALYSIS OF THREATEN DEGREE

So, the ranking result of the threaten degree of target is:

$$
x_{7}>x_{2}>x_{1}>x_{6}>x_{10}>x_{8}>x_{5}>x_{9}>x_{4}>x_{3}
$$

\section{CONCLUSION}

Aiming at the multi batch target of the terminal defense system, the article put forward a kind of improved grey comprehensive relational analysis method. Through the analysis of the example, we prove that the method is easy to implement and the evaluation result is very well. It can help the commander to make a quick command decision accurately.

\section{REFERENCES}

[1] Lebin Tan. Gun Introduction (Chapter 14).Beijing Institute of Technology press, Beijing, pp. 266-284, 2014.

[2] Yunsheng Wei \&ZhiGuo\&Xiaohui Wang.Fire Control and Command (Chapter 2).Beijing Institute of Technology press, Beijing, pp. 18-32, 2003.

[3] LihuaWeng. Grey System Theory and Its Application, Science Press, Beijing, pp. 11-77, 1991.

[4] Jifang Wang. The Determine Method Of The Membership Function, Henan Science Press, Henan,6(31), pp. 8831-8832, 2000. 